Journal of Case Reports 2020;10(4):258-260

\title{
Primary Mediastinal Germ Cell Tumors
}

\author{
Caroline E. Lippe, Troy Moritz
}

General Surgery Program, UPMC Pinnacle Community Osteopathic, 4300 Londonderry Road, Harrisburg, PA 17109, United States.

\begin{abstract}
Corresponding Author:
Dr. Caroline E. Lippe

Email: caroline.e.lippe@gmail.com

This is an Open Access article distributed under the terms of the Creative Commons Attribution License (creativecommons.org/ licenses/by/3.0).
\end{abstract}

Received : July 17, 2020

Accepted : October 2, 2020

Published : December 20, 2020

\begin{abstract}
Background: Primary malignant mediastinal germ cell tumors (PMMGCT) are exceedingly rare, accounting for $1-2 \%$ of germ cell tumors. The objective of this case report is to highlight the severity and unique characteristics of this tumor. Case Report: A young, active 33 year old male presented to a community emergency department with flu-like symptoms and hemoptysis continuously for one month. Workup noted a large anterior mediastinal mass with erosion into the sternum. Biopsy revealed a non-seminomatous germ cell tumor, with primary malignant origin in the mediastinum. Chemotherapy was initiated, which the patient has tolerated well during his first few rounds of treatment. Conclusion: PMMGCTs are rare and aggressive tumors with variable response to chemotherapy. Extensive surgery has been offered for those with resistant factors, with more complications.
\end{abstract}

Keywords: Biopsy, Germ Cell and Embryonal Neoplasms, Mediastinal Neoplasms, Nonseminomatous germ cell tumor, Neoplasms.

\section{Introduction}

Primary germ cell tumors of the mediastinum are $1-2 \%$ of all germ cell tumors and $1-3.5 \%$ of mediastinal tumors, making it an exceedingly rare find [1]. Germ cell cancers are the most common malignancy found in 15-35 year old men with 5\% of malignant tumors being extra-gonadal [2]. Gonadal and extra-gonadal tumors share similarities in that they are both found in young adult men, usually in the midline locations and are histologically found to be seminomatous or nonseminomatous with evidence of high $\beta$-HCG and AFP tumor markers. Studies indicate an elevated AFP and/or elevated $\beta$-HCG is diagnostic of a primary mediastinal non seminomatous germ cell tumor (PMNSGCT) and there is no need for testicular ultrasound or biopsy [3]. They also have a gain of isochromosome 12p, much like gonadal tumors. Some defining differing characteristics of extra-gonadal tumors are that they have increased tumor burden at initial presentation with a primary location in the anterior mediastinum and are often non-seminomatous [2]. Rare retroperitoneal lymphadenopathy is found at presentation for primary extra-gonadal tumors, as this would indicate more of a primary testicular cancer with metastatic location to the mediastinum. The idea is that extra-gonadal tumors follow the same process as development as primary gonadal tumors but the key difference is that the midline migration of yolk sac endoderm does not follow its usual pattern, therefore allowing primordial germ cells to locate in sites outside of the gonads. Common locations of the midline include the mediastinum followed by the retroperitoneum with rare findings in the pineal and presacral spaces [3]. Presenting symptoms include dyspnea, chest pain, cough, fever, weight loss, vena cava syndrome and fatigue.

\section{Case Report}

A 33 year old non-smoking man presented to the emergency department in the winter of 2020 with 
flu-like symptoms and hemoptysis for 1 month. He endorses fever, chills and $10 \mathrm{lb}$ weight loss. He was sent into the emergency department from an urgent care for an abnormal chest x-ray after being worked up for bronchitis. He underwent CT chest, showing large anterior mediastinal mass $13 \times 9.5 \times 8.9 \mathrm{~cm}$, extending towards the left upper lobe [Fig.1]. Mass is also shown to affect the left main pulmonary artery with bony destruction of the left side of the manubrium of the sternum with the tumor appearing to extend into the left anterior chest wall through the $1^{\text {st }}$ left intercostal space anteriorly. The biopsy of the anterior mass was obtained by intervention radiology. Ultrasound showed diffusely abnormal testis, with hyper-vascularization and numerous and diffuse calcifications present throughout both testes [Fig.2]. CT of the abdomen and pelvis was negative for lymphadenopathy or metastatic disease. Tumor markers indicated lactate dehydrogenase (LDH) 1,691 (140-280 U/L), human chorionic gonadotropin $(\beta-\mathrm{HCG}) 0.6(<15$ $\mathrm{ng} / \mathrm{mL}$ ) and alpha-fetoprotein (AFP) 5.3 (10-20 ng/ $\mathrm{mL})$. Biopsy of mediastinal mass resulted in a nonseminomatous germ cell tumor, likely embryonal carcinoma. Immunohistochemical staining was positive for CD30 and PLAP. He was started on chemotherapy with bleomycin, cisplatin, and etoposide.

\section{Discussion}

Primary mediastinal EGGCTs as described above, are exceedingly rare, but have a well-defined clinical presentation. The outcome of these tumors, especially if found to be non-seminomatous, are usually short-lived and have no current management guidelines. CT scan is primary modality of diagnosis followed by biopsy. Our patient did not have testicular biopsies performed at the time of publication. Patients with both seminomatous and non-seminomatous EGGCTs are found to have a risk of metachronous testicular tumors, about $10 \%$ in 10 years, showing no indication for surveillance and repeat biopsies [4]. The increased risk of leukemia is only found in primary mediastinal

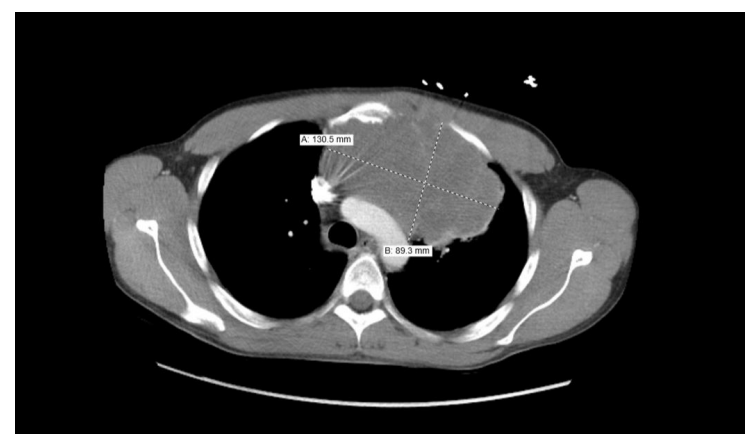

Fig.1: $C T$ chest, coronal view, showing the anterior mediastinal mass with dimensions $13 \times 9.5 \times 8.9 \mathrm{~cm}$. Noted is some erosion into the manubrium.

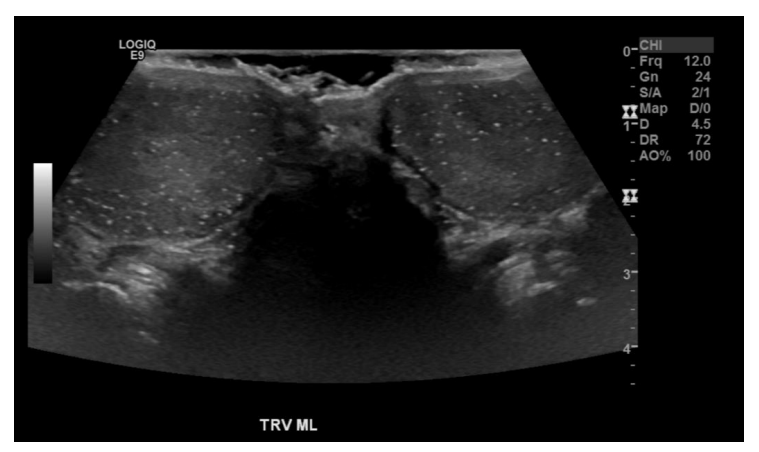

Fig.2: Ultrasound of the bilateral testes, showing multifocal disease with diffuse calcifications. No mass noted on exam.

malignant germ cell tumors (PMMGCT) of nonseminomatous origin.

The 5-year survival of non-seminomatous PMMGCTs is $45 \%$, compared to $90 \%$ for seminomatous and subsequently are deemed having a poor prognosis [5]. The old recommendation is 4 cycles of cisplatin, etoposide and bleomycin (PEB) chemotherapy, but has shown side effects of worsening pulmonary function and other pulmonary complications. Recent data has suggested use of four cycles of the VIP regime, etoposide (VePesid), ifosfamide, and cisplatin (Platinol), chemotherapy regime over PEB [3]. The benefit of VIP is that patients may need to undergo extensive secondary surgical thoracic resection for residual tumor, so decreasing pulmonary complications is beneficial [3]. Surgery after chemotherapy is essential to remove chemotherapy-resistant tumor, directly visualize response and direct subsequent 
chemotherapy. Salvage chemotherapy was found to have little to no use. Follow up after surgery includes trending tumor markers $\mathrm{AFP} / \beta-\mathrm{HCG}$ then CT scan every 2 months for the first year then every 4 months for the second year and every 6 months for 3-5 years if residual tumor was noted to have necrosis [3]. Germ cell cancer in the surgical resection indicates two post-operative courses of etoposide and cisplatin (EP). PMMGCTs are more aggressive than its gonadal counterparts with a worse prognosis. The resistance to chemotherapy, extensive disease at diagnosis and extensive surgical intervention needed without complete removal is one of the many factors leading to its lethality. Due to its genetic and histologic manifestations, it also causes an increase in other types of cancers, elevating its already poor outcome.

\section{Conclusion}

PMMGCTs are exceedingly rare and aggressive, making its diagnosis and treatment difficult. Responses to chemotherapy including eptoposide and cisplatin are promising, but resistance to chemotherapy has been shown, creating the need for more effective treatments. Extensive surgery has been offered for those with resistant factors, with more complications.

Contributors: CEL: Drafting the work and patient management; TM: substantial contributions to the conception and critical review of the work. CEL will act as a study guarantor. Both authors approved the final version of the manuscript and are responsible for all aspects of the study.

Funding: None; Competing interests: None stated.

\section{References}

1. Lemarie E, Assouline P, Diot P, Regnard JF, Levasseur $\mathrm{P}$, Droz JP, et al. Primary mediastinal germ cell tumors; Results of a French retrospective study. Chest. 1992;102:1477-1483.

2. Schmoll HJ. Extragonadal germ cell tumors. Ann Oncol. 2002;13:265-272.

3. Albany C, Einhorn LH. Extragonadal germ cell tumors: Clinical presentation and management. Curr Opin Oncol. 2013;25:261-265.

4. Hartmann JT, Fossa SD, Nichols CR, Droz JP, Horwich A, Gerl A, et al. Incidence of metachronous testicular cancer in patients with extragonadal germ cell tumors. J Natl Cancer Inst. 2001;93:1733-1737.

5. Mishra S, Das Majumdar SK, Sable M, Parida DK. Primary malignant mediastinal germ cell tumors: A single institutional experience. South Asian J Cancer. 2020;9:27-29. 